Section 5 .

OBSERVATIONAL PROJECTS 


\title{
Observational Model for Precision Astrometry with the Space Interferometry Mission
}

\author{
Slava G. Turyshev and Mark H. Milman \\ Jet Propulsion Laboratory, California Institute of Technology, \\ Pasadena, CA 91109
}

\begin{abstract}
The basic astrometric observable of the Space Interferometry Mission (SIM) instrument is the pathlength delay. This measurement is made by a combination of internal metrology measurements that determine the distance the starlight travels through the two arms of the interferometer and a measurement of the white light stellar fringe to find the point of equal pathlength. Because this operation requires a nonnegligible integration time, the interferometer baseline vector is not stationary over this time period, as its absolute length and orientation are time-varying. This paper addresses how the time-varying baseline can be "regularized" so that it may act as a single baseline vector for multiple stars, as required for the solution of the astrometric equations.
\end{abstract}

\section{Introduction}

SIM is a space-based $10-\mathrm{m}$ baseline Michelson optical interferometer operating in the visible waveband that is designed to achieve astrometric accuracy in the single digits of the microarcsecond domain. Over a narrow field of view SIM is expected to achieve a mission accuracy of $1 \mu$ as. In this mode SIM will search for planetary companions to nearby stars by detecting the astrometric "wobble" relative to a nearby $\left(\leq 1^{\circ}\right)$ reference star. In its wide-angle mode, SIM will provide $4 \mu$ as precision absolute position measurements of stars, with parallaxes to comparable accuracy, at the end of its 5-year mission. The expected proper motion accuracy is around $3 \mu \mathrm{as} / \mathrm{yr}$, corresponding to a transverse velocity of $10 \mathrm{~m} / \mathrm{s}$ at a distance of $1 \mathrm{kpc}$.

SIM surveys the sky in units called tiles. A tile is defined as a sequence of measured delays corresponding to multiple objects all made by a single baseline vector $\vec{b}$ and central pointing of the instrument - that is, all the measurements in a tile are from objects that are within a single astrometric field of regard of the instrument, which is $15^{\circ} \times 15^{\circ}$. The existence of a single baseline vector ensures that the system of equations developed from the observations to extract the astrometric parameters is not underdetermined. However, the collection of such a measurement set with a single interferometer is actually impossible, as the data collection on a sequence of objects takes finite time, over which both the baseline length and orientation do not remain constant.

This paper describes the fundamental steps of how the on-board instrumentation of external metrology and auxiliary guide interferometers are used to 
reconstruct the baseline vector sufficiently accurately so that it can effectively be modeled as a single vector over the period of a tile observation. This process has been previously referred to as the regularization of the baseline (Boden, 1997). The notion of the regularized baseline has been used extensively in a number of grid simulation studies that plan observation sequences, predict mission accuracy, and determine sensitivities to various instrument parameters (Loiseau and Malbet, 1996; Boden, 1997; Swartz, 2000).

\section{The logic of astrometry with SIM}

SIM will measure the pathlength delay between the two arms of the interferometer. The instantaneous delay is given formally by the interferometer astrometric equation:

$$
d(t)=\left(\vec{b}(t) \cdot \vec{s}_{j}\right)+k
$$

where $d$ is the optical pathlength delay that is measured by the interferometer using internal metrology and the white light fringe, $\vec{s}_{j}$ is the unit 3 -vector to the observed $j^{\text {th }}$ star, $\vec{b}$ is the baseline 3 -vector, and $k$ is a so-called constant (or calibration) term that represents possible optical path differences between the light collected from the target object and the internal metrology, and $(\vec{a} \cdot \vec{b})$ denotes the dot vector product of vectors $\vec{a}$ and $\vec{b}$. To differentiate between the various interferometer baselines we will use the notation $\vec{b}_{g A}, A \in(1,2)$, to denote the baselines of guide interferometers 1 and 2 with corresponding delay measurements $d_{\mathrm{g}_{1}}$ and $d_{\mathrm{g}_{2}}$, and $\vec{b}_{\mathbf{s}}$ will denote the baseline of the science interferometer.

Because a finite integration time is required to make measurement (1), the actual instrument measurement is:

$$
\left\langle d_{\mathbf{s}}\right\rangle=\left(\vec{s}_{j} \cdot\langle\vec{b}\rangle\right)+\left\langle k_{s}\right\rangle,
$$

where $\left\langle d_{B}\right\rangle$ is the average external delay, $\langle\vec{b}\rangle$ is the average baseline vector over the period of the observation, and $\left\langle k_{s}\right\rangle$ the same for the calibration term. Note that all of the quantities on the right are unknown, and thus must be estimated.

Because the three interferometer baselines are not colinear, to complete the characterization of the rigid body behavior of the instrument a third inertial measurement is required. This measurement is termed the "roll" measurement. In the ideal case the collection of measurements made by the guide interferometers, the roll measurement, the external metrology measurements together with the a priori data consisting of the positions of the guide stars, the initial guide and science baseline vectors in the local frame, uniquely determine the baseline vector of the science interferometer in inertial space.

We will now get into the details of how $\langle\vec{b}\rangle$ is obtained from the measurements and a priori data. For this purpose it suffices to treat SIM as a set of fiducials, $\vec{X}_{1}, \ldots, \vec{X}_{N}, \vec{X}_{i} \in \mathbf{R}^{3}$. Our interests center around the evolution of the fiducials $\vec{X}_{i}(t)$ over a time period $t_{0} \leq t \leq T$, where $t=t_{0}$ denotes the beginning of an observation of a tile and $t=T$ is the time of completion. The problem is solved using the on-board optical sensing systems that include the external 
metrology system, the guide star interferometers, and the roll estimator. The signals from these systems are briefly described next.

The observed variables associated with the external metrology system are

$$
\ell_{i j}(t)=\left|\vec{X}_{i}(t)-\vec{X}_{j}(t)\right|-\left|\vec{X}_{i}\left(t_{0}\right)-\vec{X}_{j}\left(t_{0}\right)\right|, \quad 1 \leq i \leq 4, \quad j>i .
$$

These measurements are relative distance measurements, and (3) is valid for any choice of coordinate frame (relativistic terms are not included at this point). In particular, $\vec{b}_{\mathrm{s}}$ is determined in the local frame from (3). The problem is to find $\vec{b}_{\mathrm{s}}$ in the inertial frame. This connection is made by using the guide interferometers.

SIM uses a pair of guide interferometers to produce two independent delay measurements per observation. The observed variables associated with the guide interferometers are the delays $d_{\mathrm{gA}}$ given by Eq. (1).

The roll estimator produces a "measurement" similar to the guide interferometers. Let $\vec{n}$ denote the line-of-sight vector of one of the guide interferometer telescopes and let $\vec{\tau}$ denote the baseline of the role estimator (see Milman \& Turyshev, 2000). The roll estimator equation is

$$
\kappa=(\vec{n} \cdot \vec{\tau}),
$$

where $\kappa$ is constant for all values of $\vec{n}$ and $\vec{\tau}$ over the period of a tile.

Now let $\hat{U}$ define the transformation between the local and inertial coordinate frames. Since the science baseline vector $\vec{b}_{\mathbf{s}}$ is known in local coordinates from external metrology measurements, the problem of determining $\vec{b}_{\mathbf{B}}$ in inertial coordinates is solved once we obtain $\hat{U}, v i z . \vec{b}_{\mathrm{s}}=\hat{U} \vec{b}_{\mathbf{s}}^{\text {loc }}$.

The equations for obtaining $\hat{U}$ are provided by the guide interferometer measurements and the roll estimator. The guide measurements and the roll estimator may be written as

$$
d_{\mathrm{gA}}=\left(\vec{g}_{\mathrm{A}} \cdot \hat{U} \vec{b}_{\mathrm{gA}}^{\text {loc }}\right)+k_{\mathrm{gA}}, \quad \kappa=(\vec{n} \cdot \vec{\tau})=\left(\vec{n} \cdot \hat{U} \vec{\tau}^{\text {loc }}\right),
$$

with $\vec{b}_{\mathrm{gA}}^{\text {loc }}$ and $\vec{n}, \kappa$ and $\vec{\tau}^{\text {loc }}$ all assumed known from external metrology data. We now have three equations needed to determine $\hat{U}$.

\section{Astrometric modeling for SIM}

Solving (5) requires a parameterization of $\hat{U}$. The small size of the attitude changes due to attitude control error and thermal drifts suggest that is sufficient to represent $\hat{U}$ in a truncated series expansion. As a result we represent $\hat{U}$ in the series expansion for some skew-symmetric matrix $\hat{S}$,

$$
\hat{U} \approx \hat{I}+\hat{S}+\hat{S}^{2} / 2+\mathcal{O}\left(\hat{S}^{3}\right) .
$$

The approximation Eq. (6) can be shown to solve the system (5) with an error of $\mathcal{O}\left(\left.\left|\vec{b}_{\mathbf{s}}\right||\hat{S}|\right|^{4}\right)$. Thus sub-picometer geometric accuracy for even arcmin level attitude errors, is attained. 
With this in mind, to the second order in small attitude variations, $\vec{\omega}(t)$, we parameterize the instantaneous interferometer baseline, $\vec{b}(t)$, as

$$
\vec{b}(t)=b\left(t_{0}\right)(1+\epsilon(t))\left(\vec{n}\left(t_{0}\right)+\left[\vec{\omega}(t) \times \vec{n}\left(t_{0}\right)\right]+\frac{1}{2 !}\left[\vec{\omega}(t) \times\left[\vec{\omega}(t) \times \vec{n}\left(t_{0}\right)\right]\right]\right),
$$

where $b\left(t_{0}\right), \vec{n}\left(t_{0}\right)$ are the initial baseline length and orientations of the interferometer; $\epsilon(t)=\Delta b(t) / b\left(t_{0}\right)$ are the time-varying readings of the external metrology; $\vec{\omega}(t)$ is the vector of small attitude changes in the baseline orientation for the interferometer; and $[\vec{a} \times \vec{b}]$ denotes the cross vector product.

The expression (7) allows us to write the delays for all three interferometers. Thus, to the second order in $\vec{\omega}$ one obtains the following expressions for the timevarying delays of two guide interferometers:

$$
\begin{gathered}
d_{\mathrm{gA}}(t) \equiv k_{\mathrm{gA}}(t)+b_{\mathrm{gA}}\left(t_{0}\right)\left(1+\epsilon_{\mathrm{gA}}(t)\right)\left(\vec { g } _ { \mathrm { A } } \cdot \left\{\vec{n}_{\mathrm{gA}}\left(t_{0}\right)+\left[\vec{\omega}_{\mathrm{gA}}(t) \times \vec{n}_{\mathrm{gA}}\left(t_{0}\right)\right]+\right.\right. \\
\left.\left.+\frac{1}{2 !}\left[\vec{\omega}_{\mathrm{gA}}(t) \times\left[\vec{\omega}_{\mathbf{g A}}(t) \times \vec{n}_{\mathrm{gA}}\left(t_{0}\right)\right]\right]+\mathcal{O}\left(\omega^{3}\right)\right\}\right) .
\end{gathered}
$$

Taking into account flexible body motions, and subtracting the initial conditions from the time-varying delays tracked by the guide interferometers, we define the instantaneous change in the science interferometer baseline orientation. However, the obtained system of equations is underdetermined. This is why only two out of three components of the attitude vector $\vec{\omega}_{\mathbf{s}}(t)$ may be determined this way. We call the undetermined component the roll component. For the determination of this component of the attitude vector SIM will be using the roll estimator of the form given by Eq. (5).

A solution to the obtained set of non-linear equations may be obtained in an iterative way. In consideration of brevity we present here only the first order solution for the science interferometer instantaneous delay:

$$
\begin{aligned}
& d_{\mathbf{s}}(t)=k_{0 \mathbf{s}}+\Delta k_{\mathbf{s}}(t)+b_{0}\left(1+\epsilon_{\mathbf{s}}(t)\right)\left\{\left(\vec{n}_{0} \cdot \vec{s}_{j}\right)-\right. \\
& \left.-\left(p_{\mathbf{g} 1}(t) \frac{\left(\vec{n}_{0} \cdot\left[\vec{g}_{2} \times \vec{s}_{j}\right]\right)}{\left(\vec{n}_{0} \cdot\left[\vec{g}_{1} \times \vec{g}_{2}\right]\right)}-p_{\mathbf{g} 2}(t) \frac{\left(\vec{n}_{0} \cdot\left[\vec{g}_{1} \times \vec{s}_{j}\right]\right)}{\left(\vec{n}_{0} \cdot\left[\vec{g}_{1} \times \vec{g}_{2}\right]\right)}\right)+\mathcal{O}\left(\omega_{\mathbf{s}}^{2}\right)\right\}
\end{aligned}
$$

where $p_{\mathrm{gA}}(t)$ characterizes the pathlength feed-forward signal (instrumental drifts) and has the form:

$$
p_{\mathrm{gA}}(t)=\left(1-\epsilon_{\mathrm{gA}}(t)\right) \frac{\Delta d_{\mathrm{gA}}(t)-\Delta k_{\mathrm{gA}}(t)}{b_{\mathrm{gA}}\left(t_{0}\right)}-\epsilon_{\mathrm{gA}}(t)\left(\vec{n}_{0} \cdot \vec{g}_{\mathrm{A}}\right) .
$$

We also defined the following notations: $d_{\mathbf{s}}(t), d_{\mathrm{gA}}(t)$ are the instantaneous interferometric delays; $\epsilon_{\mathbf{s}}(t), \epsilon_{\mathbf{g A}}(t)$ are the fractional baseline lengths variations; $k_{0 \mathrm{~s}}$ is the calibration term of the science interferometer at the beginning; $\Delta k_{\mathbf{s}}(t), \Delta k_{\mathrm{gA}}(t)$ are the contributions of temporal drifts in the calibration terms; $\vec{\omega}_{\mathrm{OgA}_{\mathrm{A}}}$ is the initial misalignment of the $\mathrm{A}^{\text {th }}$ guide interferometer baseline's orientation relative to that of the science interferometer, namely $\vec{n}_{\mathrm{gA}}\left(t_{0}\right)=\vec{n}_{\mathbf{s}}\left(t_{0}\right)+\left[\vec{\omega}_{\mathrm{OgA}}\left(t_{0}\right) \times \vec{n}_{\mathbf{s}}\left(t_{0}\right)\right]+\mathcal{O}\left(\omega_{0 \mathrm{gA}}^{2}\right) ; \vec{\theta}_{\mathrm{gA}}(t)$ is the change in the guide 
interferometer's orientation relative to the science baseline in the local frame, $\vec{\omega}_{\mathrm{gA}}(t)=\vec{\omega}_{\mathrm{B}}(t)+\vec{\theta}_{\mathrm{gA}}(t)$. For detailed derivation of terms $\sim \mathcal{O}\left(\omega_{\mathrm{B}}^{3}\right)$, see Milman $\&$ Turyshev (2000).

\section{Baseline regularization concept}

The process of reconstructing the baseline vector when implemented onboard in real-time is termed pathlength feedforward, and is a critical component to the operation of the interferometer. The pathlength feedforward signal is exact when the parameters that define the mapping are without error. However, $a$ priori errors in the guide star positions, the relative positions of the fiducials in the metrology local frame, and the interferometer constant terms will produce a residual error between the science baseline vector in inertial space and its estimate as derived by the feedforward algorithm.

To characterize the impact of this error on the astrometric equations, first recall that the science interferometer makes the measurement $\left\langle d_{\mathbf{s}}\right\rangle$, Eq. (2). Next let $\hat{\vec{b}}(t)$ denote the estimate of $\vec{b}(t)$ using the aforementioned measurements and a priori data. Then we write the above as

$$
\left\langle d_{\mathbf{s}}\right\rangle=\left(\vec{s}_{j} \cdot\langle\hat{\vec{b}}\rangle\right)+\left(\vec{s}_{j} \cdot(\langle\vec{b}\rangle-\langle\hat{\vec{b}}\rangle)\right)+\left\langle k_{s}\right\rangle
$$

Ideally, in the absence of measurement and a priori data errors, the second term above is zero, which is not the case in general. The important result is that to first order, as long as the guide interferometers are locked, the difference $(\langle\vec{b}\rangle-\langle\hat{\vec{b}}\rangle)$ is a constant vector. Hence, over the observation of a tile, there is a just a single vector correction to the time-varying baseline vector that can be used for all the science targets in the tile. This correction vector is the "regularization" of the baseline. The set of formulae described herein will serve as the kernel for the future mission analysis and simulations. A complete characterization and justification of these statements will appear elsewhere.

Acknowledgments. This work was performed by the Jet Propulsion Laboratory, California Institute of Technology, under contract with the National Aeronautics and Space Administration.

\section{References}

Boden, A. F., 1997, SIM Astrometric Grid Simulation Development and Performance Assessment, JPL Interoffice Memorandum.

Loiseau, S., Malbet, F., 1996, "Global astrometry with OSI," Astron. Astrophys. Sup., 116, 373-380.

Swartz, R., 2000, Metrology Breaks and the SIM Astrometric Grid, JPL Interoffice Memorandum.

Milman, M. H. and Turyshev, S. G., 2000, Observational Model for Precision Astrometry with the Space Interferometry Mission, in Proc. "Interferometry in Optical Astronomy," P. J. Lena and A. Quirrenbach, eds. , SPIE 4006, paper \#99, to be published. 